\title{
Relation of Adiponectin to Glucose Tolerance Status, Adiposity, and Cardiovascular Risk Factor Load
}

\author{
N. Wolfson, ${ }^{1}$ D. Gavish, ${ }^{1}$ Z. Matas, ${ }^{2}$ M. Boaz, ${ }^{3}$ and M. Shargorodsky ${ }^{4}$ \\ ${ }^{1}$ Department of Medicine, Edith Wolfson Medical Center and Sackler School of Medicine, Tel Aviv University, Tel Aviv 69978, Israel \\ ${ }^{2}$ Department of Biochemistry, Edith Wolfson Medical Center, Holon 58100, Israel \\ ${ }^{3}$ Epidemiology and Research Unit, Edith Wolfson Medical Center and Sackler School of Medicine, Tel Aviv University, \\ Tel Aviv 69978, Israel \\ ${ }^{4}$ Department of Endocrinology, Edith Wolfson Medical Center and Sackler School of Medicine, Tel Aviv University, \\ P. O. Box 5, Holon 58100, Israel
}

Correspondence should be addressed to M. Shargorodsky, marinas@wolfson.health.gov.il

Received 17 July 2011; Accepted 10 November 2011

Academic Editor: K. Khunti

Copyright ( 2012 N. Wolfson et al. This is an open access article distributed under the Creative Commons Attribution License, which permits unrestricted use, distribution, and reproduction in any medium, provided the original work is properly cited.

\begin{abstract}
Objective. Adiponectin has anti-atherogenic and anti-inflammatory properties. We investigated the influence of adiponectin on glucose tolerance status, adiposity and cardiovascular risk factors (CVRFs). Design and Patients. Study consisted of 107 subjects: 55 with normal glucose tolerance (NGT) and 52 with impaired glucose regulation (IGR) who were divided into two groups: 24 subjects with impaired fasting glucose (IFG Group) and 28 patients with type 2 diabetes mellitus (DM Group). In additional analysis, study participants were divided into two groups, according to CVRFs: low and high risk. Measurements: Patients were evaluated for glucose, HbA1C, insulin, lipids, CRP, HOMA-IR and adiponectin. Measurements. Patients were evaluated for glucose, HbA1C, insulin, lipids, CRP, HOMA-IR and adiponectin. Results. Adiponectin was significantly higher in NGT group than in IFG $(P=$ $0.003)$ and DM $(P=0.01)$ groups. Adiponectin was significantly, positively associated with HDL and inversely associated with glucose, HbA1c, ALT, AST, TG, HOMA-IR. Patients with higher CVRFs load have lesser adiponectin compared to patients with low cardiovascular risk $P<0.0001)$. Adiponectin was inversely associated with the number of risk factors $(r=-0.430, P=0.0001)$. Conclusions. Circulating adiponectin was significantly lower in subjects with different degree of IGR compared to subjects with normal glucose homeostasis. Adiponectin was significantly lower in high risk group than low risk group and decreased concurrently with increased number of CVRFs.
\end{abstract}

\section{Introduction}

Adiponectin, a collagen-like protein specifically and highly expressed in human adipose cells, plays an important role in insulin sensitivity, inflammation, lipid metabolism, and atherogenesis [1-3]. Low plasma adiponectin levels are significantly correlated with endothelial dysfunction, increased intima media thickness, and progression of coronary artery calcification independently of other cardiovascular risk factors [4-6]. Plasma adiponectin levels are reduced not only among obese patients but also in disease states frequently associated with obesity, such as type 2 diabetes, hypertension, metabolic syndrome, and coronary artery disease [7-9]. Altogether, these data suggest that adiponectin may mediate its effects via obesity-independent mechanisms, but whether obesity per se or other pathways play a regulatory role is unclear and deserves further evaluation. The present study was designed to assess the relation between plasma adiponectin levels to glucose tolerance status, adiposity, and cardiovascular risk factors (CVRFs) load.

\section{Methods}

The study group consisted of 107 Caucasian subjects, (67 females, mean age $56.4 \pm 10.0$ years) who were recruited from the outpatient metabolic clinic and evaluated for the study. The study participants were divided into three groups according to glucose tolerance status: 55 with normal glucose 
TABLE 1: Clinical characteristics of study patients by glucose tolerance status.

\begin{tabular}{|c|c|c|c|}
\hline Variables & $\begin{array}{c}\text { NGT group } \\
n=55\end{array}$ & $\begin{array}{l}\text { IFG group } \\
n=24\end{array}$ & $\begin{array}{c}\text { DM group } \\
n=28\end{array}$ \\
\hline Female/male & $39 / 16$ & $7 / 17$ & $17 / 11$ \\
\hline Age $(y)$ & $55.7 \pm 9.5$ & $58.8 \pm 9.6$ & $55.9 \pm 11.3$ \\
\hline BMI $\left(\mathrm{kg} / \mathrm{m}^{2}\right)$ & $33.3 \pm 6.5$ & $32.9 \pm 6.8$ & $31.6 \pm 6.2$ \\
\hline Current smokers, $n(\%)$ & $7(13 \%)$ & $3(8 \%)$ & $3(11 \%)$ \\
\hline Hypertension, $n(\%)$ & $28(52 \%)$ & $10(42 \%)$ & $15(54 \%)$ \\
\hline Dyslipidemia, $n(\%)$ & $24(44 \%)$ & $12(50 \%)$ & $17(61 \%)$ \\
\hline Systolic blood pressure $(\mathrm{mmHg})$ & $131.5 \pm 18.4$ & $134.7 \pm 18.7$ & $134.9 \pm 19.6$ \\
\hline Diastolic blood pressure $(\mathrm{mmHg})$ & $73.4 \pm 14.1$ & $76.5 \pm 12.1$ & $76.0 \pm 9.6$ \\
\hline Fasting glucose $(\mathrm{mg} / \mathrm{dL})$ & $89.5 \pm 7.3$ & $109.4 \pm 7.6^{*}$ & $156.0 \pm 53.5^{*}$ \\
\hline HBAIC (\%) & $5.7 \pm 0.4$ & $5.9 \pm 0.5^{*}$ & $8.0 \pm 2.1^{*}$ \\
\hline HDL-cholesterol (mg/dL) & $56.6 \pm 21.3$ & $47.6 \pm 13.4^{*}$ & $45.9 \pm 18.3^{*}$ \\
\hline Triglycerides (mg/dL) & $119.9 \pm 59.4$ & $150.6 \pm 62.0^{*}$ & $195.3 \pm 158.4^{*}$ \\
\hline Hs-CRP (mg/dL) & $0.9 \pm 0.4$ & $0.7 \pm 1.2$ & $0.9 \pm 1.1$ \\
\hline $\operatorname{ALT}(\mathrm{mg} / \mathrm{dL})$ & $26.2 \pm 12.8$ & $30.9 \pm 16.2$ & $33.6 \pm 29.1^{*}$ \\
\hline $\operatorname{AST}(\mathrm{mg} / \mathrm{dL})$ & $22.9 \pm 7.8$ & $27.5 \pm 8.6$ & $28.0 \pm 18.0$ \\
\hline Fasting insulin (IU/mL) & $12.6 \pm 7.2$ & $26.0 \pm 20.1^{*}$ & $18.8 \pm 16.0^{*}$ \\
\hline HOMA-IR & $2.8 \pm 1.7$ & $7.2 \pm 5.7^{*}$ & $7.2 \pm 7.6^{*}$ \\
\hline Adiponectin (ng/mL) & $12597.2 \pm 7237.6$ & $7567.5 \pm 4193.5^{*}$ & $7484.2 \pm 4563.9^{*}$ \\
\hline
\end{tabular}

${ }^{*} P$ value versus NGT group (significant at the 0.05 level).

tolerance (NGT Group), 24 subjects with impaired fasting glucose (IFG Group), and 28 patients with type 2 diabetes mellitus (DM Group).

The cutoff for IFG was defined as FBG $=100-125 \mathrm{mg} /$ $\mathrm{dL}$, according to current ADA criteria [10].

In additional analysis study, participants were divided into two groups, according to CVRFs: low- and high-risk subjects. Cardiovascular risk factors were defined using the ATP III definition and included the following disorders: hypertriglyceridemia $(\geq 150 \mathrm{mg} / \mathrm{dL}$ and/or pharmacological treatment), low HDL cholesterol level $(<40 \mathrm{mg} / \mathrm{dL}$ in men and $<50 \mathrm{mg} / \mathrm{dL}$ in women), high blood pressure (systolic blood pressure $\geq 130 \mathrm{mmHg}$ and/or diastolic $\mathrm{BP} \geq 85 \mathrm{mmHg}$ and/or pharmacological treatment), and elevated plasma glucose $\geq 100 \mathrm{mg} / \mathrm{dL}$ and/or pharmacological treatment). Obesity was defined using the World Health Organization criteria (BMI $\geq 30 \mathrm{~kg} / \mathrm{m}^{2}$ ). According to the above-mentioned CVRFs, the high-risk group included patients with three or more CVRFs $(n=55)$, whereas low risk had two or less CVRFs $(n=52)$.

2.1. Biochemical Parameters. Blood sampling for full chemistry and metabolic parameters, including fasting glucose, HbA1C, c-peptide, insulin, lipids profile, fibrinogen, hs-CRP, and plasma adiponectin, will be performed. Adiponectin will be determined by a commercial sandwich enzyme immunoassay technique, R\&D Systems, Minneapolis, MN, USA (catalog number DRP300) with 2.8\% intra-assay and 6.5\% interassay variability.

Insulin resistance and $\beta$-cell function was estimated using homeostasis model assessment. Homeostasis model assessment-insulin resistance (HOMA-IR) will be calculated by the following formula: fasting plasma insulin $(\mathrm{mU} / \mathrm{mL}) \times$ fasting plasma glucose $(\mathrm{mg} / \mathrm{dL}) / 405$ [11], HOMA for $\beta$-cell function $(\mathrm{HOMA}-\beta)$ as follows: $20 \times$ fasting plasma insulin $(\mathrm{mU} / \mathrm{mL}) /$ fasting plasma glucose $(\mathrm{mmol} / \mathrm{L})-3.5$.

2.2. Statistical Analysis. Analysis of data was carried out using SPSS 9.0 statistical analysis software (SPSS Inc., Chicago, IL, USA, 1999). For continuous variables, such as hemodynamic and biochemistry measures, descriptive statistics were calculated and reported as mean \pm standard deviation. Distributions of continuous variables were assessed for normality using the Kolmogorov-Smirnov test (cutoff at $P=$ 0.01 ). Associations between continuous variables with approximately normal distributions including anthropometric, metabolic, and hemodynamic parameters were described using Pearson's correlation coefficients. Associations between continuous variables with distributions significantly deviating from normal were described using Spearman's rho coefficients. All tests are twosided and considered significant at $P<0.05$.

\section{Results}

Demographic and clinical characteristics of the study groups according to glucose tolerance status are presented in Table 1. As can be seen, all groups were similar in terms of age, $\mathrm{BMI}$, and presence of concomitant cardiovascular risk factors such as hypertension, dyslipidemia, and smoking. Fasting glucose differed significantly between each group and every other group, lowest in NGT subjects and highest in patients with DM, as expected. HOMA-IR as well as fasting insulin was significantly lower in NGT group than in IFG and DM 
TABLE 2: Correlations.

\begin{tabular}{|c|c|c|}
\hline \multirow{3}{*}{ Age } & \multicolumn{2}{|c|}{ Circulating adiponectin } \\
\hline & $r$-value & $0.295(* *)$ \\
\hline & $P$ value & 0.002 \\
\hline \multirow{2}{*}{ BMI } & $r$-value & -0.008 \\
\hline & $P$ value & 0.931 \\
\hline \multirow{2}{*}{ AST } & $r$-value & $-0.279(* *)$ \\
\hline & $P$ value & 0.004 \\
\hline \multirow{2}{*}{ ALT } & $r$-value & $-0.348(* *)$ \\
\hline & $P$ value & 0.0001 \\
\hline \multirow{2}{*}{ HDL-cholesterol } & $r$-value & $0.418(* *)$ \\
\hline & $P$ value & 0.0001 \\
\hline \multirow{2}{*}{ LDL-cholesterol } & $r$-value & 0.128 \\
\hline & $P$ value & 0.206 \\
\hline \multirow{2}{*}{ Triglycerides } & $r$-value & $-0.453(* *)$ \\
\hline & $P$ value & 0.0001 \\
\hline \multirow{2}{*}{ Hs-CRP } & $r$-value & -0.169 \\
\hline & $P$ value & 0.085 \\
\hline \multirow{2}{*}{ Fasting glucose } & $r$-value & $-0.375\left(^{* *}\right)$ \\
\hline & $P$ value & 0.0001 \\
\hline \multirow{2}{*}{ HbA1C } & $r$-value & $-0.328(* *)$ \\
\hline & $P$ value & 0.001 \\
\hline \multirow{2}{*}{ Fasting insulin } & $r$-value & $-0.537\left({ }^{* *}\right)$ \\
\hline & $P$ value & 0.0001 \\
\hline \multirow{2}{*}{ HOMA-IR } & $r$-value & $-0.623(* *)$ \\
\hline & $P$ value & 0.0001 \\
\hline
\end{tabular}

** Correlation is significant at the 0.01 level (2-tailed).

groups, but not different between IFG and DM groups. Additionally, significant intergroup differences were detected for HDL-cholesterol and triglycerides.

Adiponectin was significantly higher in NGT group than in IFG $(P=0.003)$ and DM $(P=0.01)$ groups. A difference in plasma adiponectin levels between IFG and DM groups was not detected.

As shown in Table 2, adiponectin was significantly, positively associated with HDL, significantly inversely associated with glucose, HbA1c, ALT, AST, triglycerides, insulin, HOMA-IR, and marginally inversely associated with CRP. No association between adiponectin and BMI was observed $(r=-0.008, P=0.931)$. Multiple regression models which were arrived at using a backward, stepwise approach were performed to identify variables independently associated with plasma adiponectin levels. In this model, adiponectin was significantly inversely associated with $\mathrm{HbA1C}$ (standardized beta $=-0.385, P=0.009$ ) and ALT (standardized beta $=-0.453, P=0.040)$. The overall model is significant $(P<0.0001)$ and explains $38.2 \%$ of variability in plasma adiponectin levels.

In additional statistical analysis, the study participants were divided into two groups according to the presence of CVRFs: low-risk and high-risk. As can be seen in Table 3, age and BMI were not different between low-risk and high-risk patients. High risk-subjects had a median of 3 CVRFs (range
3-4), while low-risk subjects had a median of 2 CVRFs (range $0-2$ ). Systolic and diastolic blood pressure as well as fasting glucose, HbA1C, fasting insulin, HOMA-IR, triglycerides, AST, and LDL-cholesterol were significantly higher and HDL-cholesterol significantly lower in high versus low-risk patients. Plasma adiponectin levels were significantly lower in high-risk group compared to low-risk subjects (6755.6 \pm 3492.2 versus $13701.1 \pm 7051.5 \mathrm{ng} / \mathrm{mL}, P<0.0001)$.

Adiponectin was significantly inversely associated with the number of risk factors $(r=-0.430, P=0.0001)$ (Table 4$)$.

\section{Discussion}

In the present study, circulating adiponectin was significantly lower in subjects with different degree of IGR compared to subjects with normal glucose homeostasis. Furthermore we confirm previously observed positive correlations of adiponectin with HDL and negative correlations with glucose, HbAlc, triglycerides, insulin, and HOMA-IR and report an inverse correlation with ALT. In multiple regression models $\mathrm{HbA1C}$ as well as ALT was independent determinant of adiponectin levels and explained $38.2 \%$ of variability in plasma adiponectin levels. Additionally, adiponectin was significantly lower in high-risk group than low-risk group and decreased concurrently with increased number of CVRFs. Thus, hypoadiponectinemia is more intensively related to glucose intolerance and CVRFs load than to adiposity.

The site and mechanism of adiponectin actions on glucose metabolism remain unknown. Recent genome-wide scans have mapped a diabetes susceptibility locus to chromosome 3q27, where the adiponectin gene (apM1) is located [12]. Evidence of an association between type 2 diabetes and single nucleotide polymorphisms at positions 45 and 276 and in the proximal promoter and exon 3 of the adiponectin gene has been also reported [11]. Furthermore, it has been shown that circulating adiponectin levels are significantly lower in healthy first-degree relatives of type 2 diabetic patients [13].

In our study, plasma adiponectin levels were significantly lower in both IFG and DM groups compared to subjects with normal glucose tolerance. Moreover, $\mathrm{HbA1C}$ levels were independent determinant of circulating adiponectin. Therefore, there is a scientific rationale that plasma adiponectin is a target for future research in the treatment and prevention of diabetes. We suggest that increase in adiponectin levels by combination of lifestyle modifications and medications may have a beneficial effect in patients with impaired glucose regulation.

Our study confirmed previously observed strong inverse associations between low adiponectin levels and some of the well-known risk factors for atherosclerosis, such as low HDLcholesterol levels, hypertriglyceridemia, and insulin resistance [14-16]. Additionally, we found significant inverse correlation between adiponectin and ALT, a surrogate marker for nonalcoholic fatty liver disease, which has recently been considered as a predictor for development of type 2 diabetes and metabolic syndrome [17-19]. These findings clearly support the point of view that adiponectin may have an active role in the pathogenesis of cardiovascular diseases. 
TABLE 3: Clinical characteristics of study patients by CVRFs load.

\begin{tabular}{lccc}
\hline Variables & Low-risk subjects & High-risk subjects & $n=55$ \\
& $n=52$ & $55.2 \pm 10.8$ & $P$ value \\
\hline Age & $57.7 \pm 9.1$ & $27(49 \%)$ & 0.200 \\
Sex (M/F) & $13(25 \%)$ & $33.7 \pm 6.2$ & 0.01 \\
BMI & $31.8 \pm 6.7$ & 3 (range 3-4) & 0.135 \\
CVRFs median (range) & 2 (range $0-2)$ & $137.9 \pm 18.2$ & 0.002 \\
Systolic blood pressure (mmHg) & $128.1 \pm 18.0$ & $77.7 \pm 11.1$ & 0.006 \\
Diastolic blood pressure (mmHg) & $72.7 \pm 12.5$ & $99.0 \pm 14.4$ & 0.032 \\
Mean arterial pressure (mmHg) & $94.7 \pm 14.2$ & $128.2 \pm 45.8$ & 0.126 \\
Fasting blood glucose (mg/dL) & $93.7 \pm 19.0$ & $45.0 \pm 1.8$ & 15.4 \\
HBAIC (\%) & $5.7 \pm 0.6$ & $186.0 \pm 119.4$ & 0.0001 \\
HDL-cholesterol (mg/dL) & $56.5 \pm 16.1$ & $0.9 \pm 1.1$ & 0.0001 \\
Triglycerides (mg/dL) & $104.0 \pm 47.5$ & $22.6 \pm 17.4$ & 0.0001 \\
Hs-CRP (mg/dL) & $0.9 \pm 0.4$ & $7.0 \pm 4.5$ & 0.0001 \\
Fasting insulin (IU/mL) & $11.9 \pm 7.6$ & $28.0 \pm 14.3$ & 0.408 \\
HOMA-IR & $2.7 \pm 1.8$ & $32.6 \pm 23.6$ & 0.0001 \\
AST (mg/dL) & $22.4 \pm 7.0$ & $6755.6 \pm 3492.2$ & 0.0001 \\
ALT (mg/dL) & $25.6 \pm 12.1$ & 0.011 \\
Adiponectin (ng/mL) & $13701.1 \pm 7051.5$ & 0.204 \\
\hline
\end{tabular}

TABLE 4: Correlations between adiponectin levels and number of CVRFs.

\begin{tabular}{|c|c|c|c|c|}
\hline & Correlations & & CVRF's & Adiponectin \\
\hline \multirow{6}{*}{ Spearman's rho } & \multirow{3}{*}{ CVRF's } & Correlation coefficient & 1.000 & $-0.430(* *)$ \\
\hline & & Sig. (2-tailed) & & 0.0001 \\
\hline & & $\mathrm{N}$ & 107 & 107 \\
\hline & \multirow{3}{*}{ Adiponectin } & Correlation coefficient & $-0.430(* *)$ & 1.000 \\
\hline & & Sig. (2-tailed) & 0.0001 & \\
\hline & & $\mathrm{N}$ & 107 & 107 \\
\hline
\end{tabular}

** Correlation is significant at the 0.01 level (2-tailed).

In the present study, no associations of adiponectin with BMI have been found; however, other studies have been unable to detect associations between adiponectin and wholebody fat mass and subcutaneous fat volume [20]. Nevertheless, an association between BMI and circulating adiponectin levels has been reported by other authors [21, 22]. Although we did not find the associations of adiponectin with BMI as well as differences in adiponectin levels between obese (BMI $\geq 30 \mathrm{~kg} / \mathrm{m}^{2}$ ) and nonobese study participants, the possibility that the relatively small sample size in the present study limited exploration of these potential correlations cannot be excluded.

The present study observed strong associations between adiponectin levels and glucose tolerance status as well as number of cardiovascular risk factors. Thus, despite the fact that adiponectin is highly specific to adipose tissue, hypoadiponectinemia is more intensively related to impaired glucose regulation and CVRFs load than to adiposity. The findings of our study are in accordance with published data that circulating adiponectin level is a strong risk marker for metabolic syndrome independent of measures of adiposity [15]. Moreover, decreased plasma adiponectin levels are inde- pendently associated with the presence of coronary artery disease in men even after adjustment for BMI [23]. Among healthy men in the Health Professionals Follow-up Study, a doubling of adiponectin levels was associated with a $30 \%$ decreased risk for myocardial infarction after adjustment for BMI, alcohol consumption, physical activity, diabetes, and hypertension [24]. Similarly, among patients with type 1 and type 2 diabetes mellitus, increased circulating adiponectin levels were associated with a lower risk of coronary artery disease after adjusting for standard risk factors $[25,26]$. These combined findings indicate that adiponectin may have a direct antiatherogenic role or mediate its effects via obesity-independent mechanisms.

Thus, plasma adiponectin integrates with the cardiovascular risk factors and is a potential target for research in reducing morbidity and mortality of atherosclerotic disease. Future research should focus on clinical endpoints such as prevention of diabetes and reduction of cardiovascular morbidity and mortality following adiponectin increase, as well as the ability of adiponectin values to predict these outcomes. 


\section{References}

[1] N. Stefan, B. Vozarova, T. Funahashi et al., "Plasma adiponectin concentration is associated with skeletal muscle insulin receptor tyrosine phosphorylation, and low plasma concentration precedes a decrease in whole-body insulin sensitivity in humans," Diabetes, vol. 51, no. 6, pp. 1884-1888, 2002.

[2] T. Yokota, K. Oritani, I. Takahashi et al., "Adiponectin, a new member of the family of soluble defense collagens, negatively regulates the growth of myelomonocytic progenitors and the functions of macrophages," Blood, vol. 96, no. 5, pp. 17231732, 2000.

[3] N. Ouchi, S. Kihara, Y. Arita et al., "Adipocyte-derived plasma protein, adiponectin, suppresses lipid accumulation and class A scavenger receptor expression in human monocyte-derived macrophages," Circulation, vol. 103, no. 8, pp. 1057-1063, 2001.

[4] K. C. B. Tan, A. Xu, W. S. Chow et al., "Hypoadiponectinemia is associated with impaired endothelium-dependent vasodilation," Journal of Clinical Endocrinology and Metabolism, vol. 89, no. 2, pp. 765-769, 2004.

[5] M. Shargorodsky, M. Boaz, Y. Goldberg et al., "Adiponectin and vascular properties in obese patients: is it a novel biomarker of early atherosclerosis," International Journal of Obesity, vol. 33, no. 5, pp. 553-558, 2009.

[6] S. Störk, M. L. Bots, P. Angerer et al., "Low levels of adiponectin predict worsening of arterial morphology and function," Atherosclerosis, vol. 194, no. 2, pp. e147-e153, 2007.

[7] W. Koenig, N. Khuseyinova, J. Baumert, C. Meisinger, and H. Löwel, "Serum concentrations of adiponectin and risk of type 2 diabetes mellitus and coronary heart disease in apparently healthy middle-aged men. Results from the 18-year followup of a large cohort from Southern Germany," Journal of the American College of Cardiology, vol. 48, no. 7, pp. 1369-1377, 2006.

[8] Y. Iwashima, T. Katsuya, K. Ishikawa et al., "Hypoadiponectinemia is an independent risk factor for hypertension," Hypertension, vol. 43, no. 6, pp. 1318-1323, 2004.

[9] K. Hotta, T. Funahashi, Y. Arita et al., "Plasma concentrations of a novel, adipose-specific protein, adiponectin, in type 2 diabetic patients," Arteriosclerosis, Thrombosis, and Vascular Biology, vol. 20, no. 6, pp. 1595-1599, 2000.

[10] S. Genuth, K. G. Alberti, P. Bennett et al., "The expert committee on the diagnosis and classification of diabetes mellitus follow-up report on the diagnosis of diabetes mellitus," Diabetes Care, vol. 26, pp. 3160-3167, 2003.

[11] L. L. Li, X. L. Kang, X. J. Ran et al., "Associations between $45 \mathrm{~T} / \mathrm{G}$ polymorphism of the adiponectin gene and plasma adiponectin levels with type 2 diabetes," Clinical and Experimental Pharmacology and Physiology, vol. 34, no. 12, pp. 12871290, 2007.

[12] M. Stumvoll, O. Tschritter, A. Fritsche et al., "Association of the T-G polymorphism in adiponectin (Exon 2) with obesity and insulin sensitivity: interaction with family history of type 2 diabetes," Diabetes, vol. 51, no. 1, pp. 37-41, 2002.

[13] F. Pellmé, U. Smith, T. Funahashi et al., "Circulating adiponectin levels are reduced in nonobese but insulin-resistant first-degree relatives of type 2 diabetic patients," Diabetes, vol. 2, no. 5, pp. 1182-1186, 2003.

[14] Y. Yamamoto, H. Hirose, I. Saito et al., "Correlation of the adipocyte-derived protein adiponectin with insulin resistance index and serum high-density lipoprotein-cholesterol, independent of body mass index, in the Japanese population," Clinical Science, vol. 103, no. 2, pp. 137-142, 2002.
[15] J. Hung, B. M. McQuillan, P. L. Thompson, and J. P. Beilby, "Circulating adiponectin levels associate with inflammatory markers, insulin resistance and metabolic syndrome independent of obesity," International Journal of Obesity, vol. 32, no. 5, pp. 772-779, 2008.

[16] A. J. Krentz, D. V. Mühlen, and E. Barrett-Connor, "Adipocytokines, sex hormones, and cardiovascular risk factors in postmenopausal women: factor analysis of the Rancho Bernardo study," Hormone and Metabolic Research, vol. 41, no. 10, pp. 773-777, 2009.

[17] S. G. Kim, H. Y. Kim, J. A. Seo et al., "Relationship between serum adiponectin concentration, pulse wave velocity and nonalcoholic fatty liver disease," European Journal of Endocrinology, vol. 152, no. 2, pp. 225-231, 2005.

[18] B. Vozarova, N. Stefan, R. S. Lindsay et al., "High alanine aminotransferase is associated with decreased hepatic insulin sensitivity and predicts the development of type 2 diabetes," Diabetes, vol. 51, no. 6, pp. 1889-1895, 2002.

[19] K. M. Choi, J. Lee, K. W. Lee et al., "Serum adiponectin concentrations predict the developments of type 2 diabetes and the metabolic syndrome in elderly Koreans," Clinical Endocrinology, vol. 61, no. 1, pp. 75-80, 2004.

[20] L. Lenchik, T. C. Register, F. C. Hsu et al., "Adiponectin as a novel determinant of bone mineral density and visceral fat," Bone, vol. 33, no. 4, pp. 646-651, 2003.

[21] Y. Arita, S. Kihara, N. Ouchi et al., "Paradoxical decrease of an adipose-specific protein, adiponectin, in obesity," Biochemical and Biophysical Research Communications, vol. 257, no. 1, pp. 79-83, 1999.

[22] M. Matsubara, S. Maruoka, and S. Katayose, "Inverse relationship between plasma adiponectin and leptin concentrations in normal-weight and obese women," European Journal of Endocrinology, vol. 147, no. 2, pp. 173-180, 2002.

[23] M. Kumada, S. Kihara, S. Sumitsuji et al., "Coronary artery disease association of hypoadiponectinemia with coronary artery disease in men," Arteriosclerosis, Thrombosis, and Vascular Biology, vol. 23, pp. 85-89, 2003.

[24] T. Pischon, C. J. Girman, G. S. Hotamisligil, N. Rifai, F. B. $\mathrm{Hu}$, and E. B. Rimm, "Plasma adiponectin levels and risk of myocardial infarction in men," Journal of the American Medical Association, vol. 291, no. 14, pp. 1730-1737, 2004.

[25] M. B. Schulze, I. Shai, E. B. Rimm, T. Li, N. Rifai, and F. B. Hu, "Adiponectin and future coronary heart disease events among men with type 2 diabetes," Diabetes, vol. 54, no. 2, pp. 534539, 2005.

[26] T. Costacou, J. C. Zgibor, R. W. Evans et al., "The prospective association between adiponectin and coronary artery disease among individuals with type 1 diabetes. The Pittsburgh epidemiology of diabetes complications study," Diabetologia, vol. 48, no. 1, pp. 41-48, 2005. 


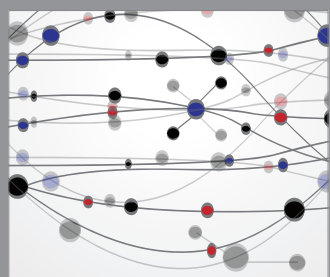

The Scientific World Journal
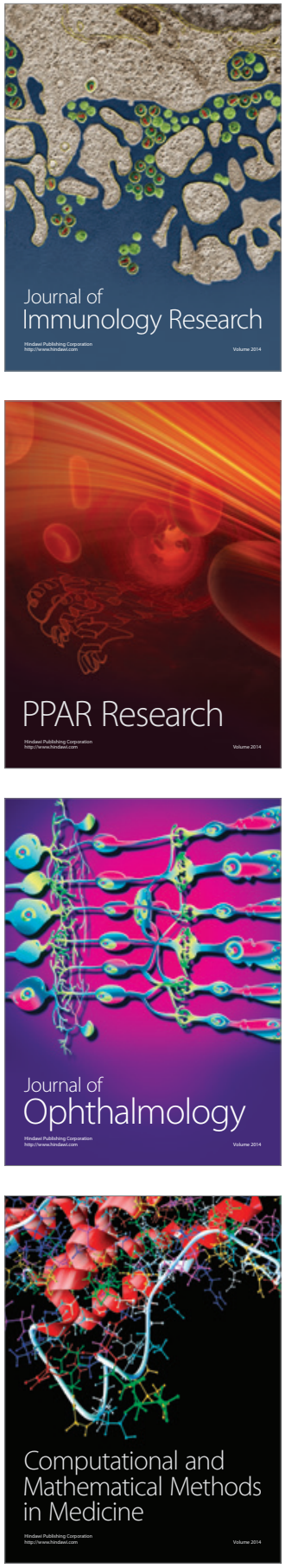

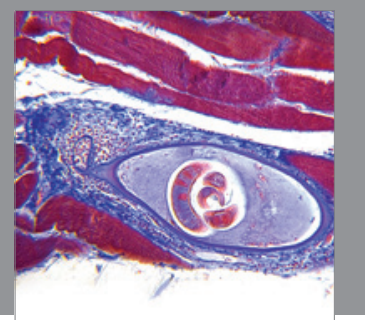

Gastroenterology

Research and Practice
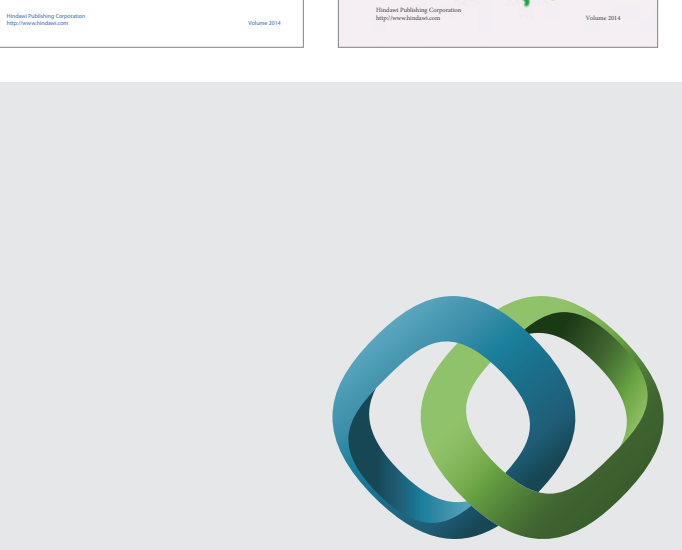

\section{Hindawi}

Submit your manuscripts at

http://www.hindawi.com
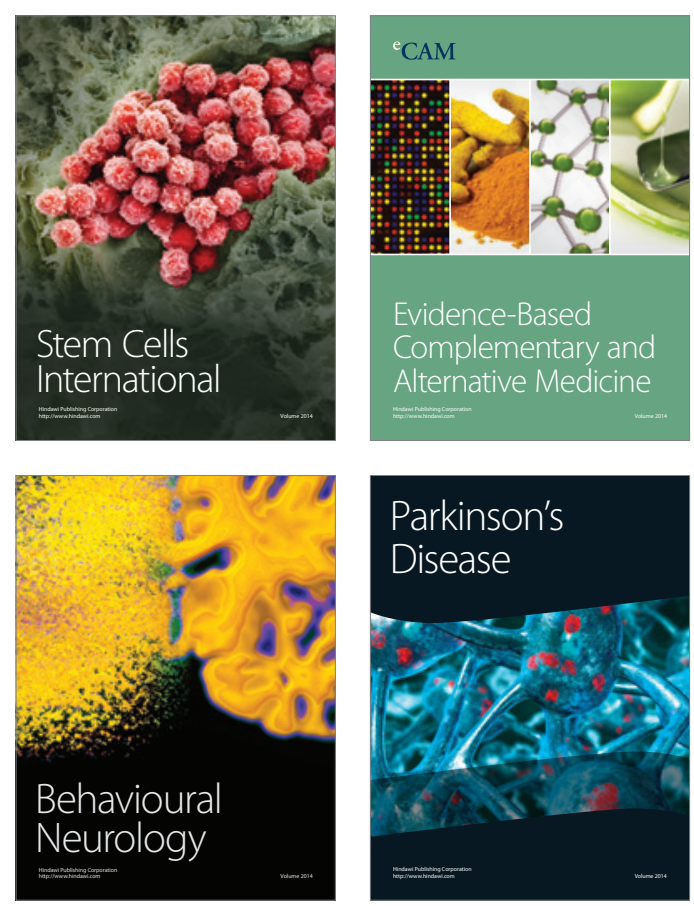

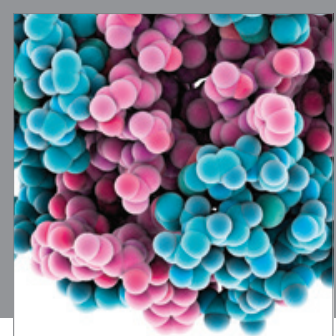

Journal of
Diabetes Research

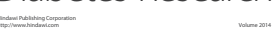

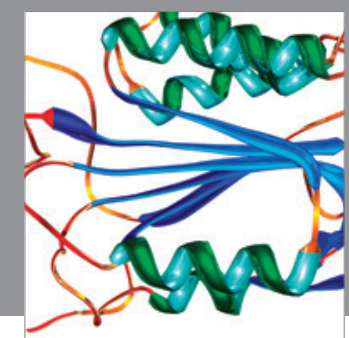

Disease Markers
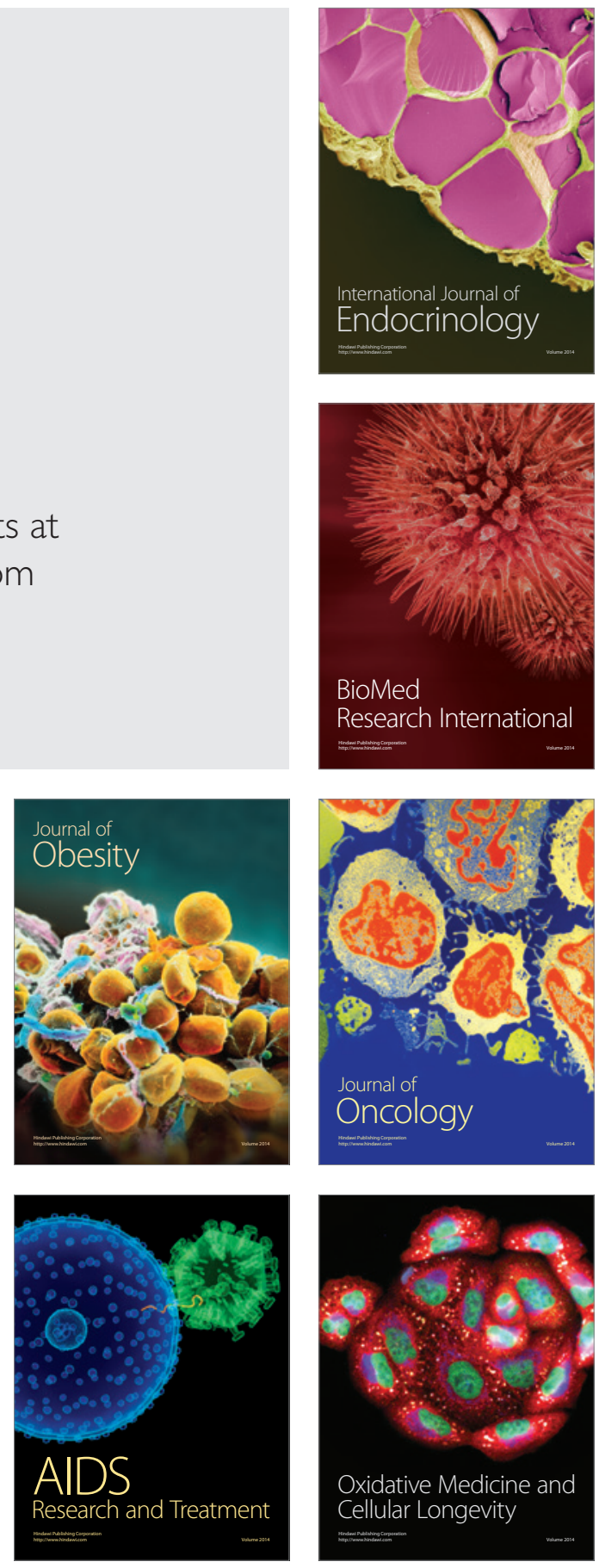